\title{
A Literature Review on Freeway Traffic Incidents and Their Impact on Traffic Operations
}

\author{
Md Ahsanul Islam \\ Department of Civil and Coastal Engineering, University of Florida, Gainesville, FL, USA \\ Email: ahsancuet08@gmail.com
}

How to cite this paper: Islam, M.A. (2019) A Literature Review on Freeway Traffic Incidents and Their Impact on Traffic Operations. Journal of Transportation Technologies, 9, 504-516.

https://doi.org/10.4236/jtts.2019.94032

Received: August 24, 2019

Accepted: October 14, 2019

Published: October 17, 2019

Copyright (c) 2019 by author(s) and Scientific Research Publishing Inc. This work is licensed under the Creative Commons Attribution International License (CC BY 4.0).

http://creativecommons.org/licenses/by/4.0/

\begin{abstract}
Congestion on the freeway is more frequent due to several traffic incidents, namely traffic accidents, debris on the road, vehicle breakdown, and collision with guardrails than any other incidents. These, in turn, affect the operational performance of the freeway by increasing queue length, volume, and density. Consequently, effective freeway management strategies can help to minimize these impacts. The study investigates and summarizes existing studies to identify the reasons for and effects of the traffic incidents. Attention is given to the available solutions of the freeway traffic incidents management. The ultimate goal of this study is to identify the gaps which are not yet addressed to improve the operational effectiveness of the freeway. This study was conducted through a comprehensive literature review of existing refereed publications, established standards, and formal guidelines. Literature was sought through the Transport Research International Documentation (TRID) database, IEEE Transactions database, and google scholar search engine. Research focusing on freeway traffic incidents is a growing concern in transportation operations, as transportation network performance depends on it. Due to the advancement of technology, emerging vehicle technologies like connected vehicles have the potential to address these problems affecting the US transportation system and revolutionize mobility in the future. The study can serve as a reference for the researchers that are involved in freeway traffic operations.
\end{abstract}

\section{Keywords}

Traffic Incidents, Freeway Incident Management, Impacts of Incidents, Operational Performance Measurement 


\section{Introduction}

Freeway traffic incidents are an important factor to estimate the performance measurement of the freeway. It disrupts the normal traffic flow pattern of the freeways. The most common form of traffic incidents are accidents, debris on the freeway, mechanical breakdown of the vehicles, and roadside fires [1] [2] [3]. Moreover, more comprehensive definition is "incident is any non-recurring event reduces the capacity of the roadway or increases the demand. Such events include traffic crashes, disabled vehicles, spilled cargo, highway maintenance, and reconstruction projects, and special non-emergency events." [4]. The effect of the traffic incidents may vary from time to time.

Congestion can be caused by routinely traffic volume at bottleneck known as recurrent congestion or traffic incidents such as traffic accidents, debris on the freeway, and roadside fire, mechanical breakdown of the vehicles, and special events known as non-recurrent congestion. Typically, traffic accidents are the major contributors to non-recurrent congestion. Literature review reveals it constitutes approximately $60 \%$ of the total freeway congestion [1] [5] [6]. Hence, effective incident management strategies are essential to improve the adverse traffic conditions.

\subsection{Research Background}

Manual on Uniform Traffic Control Devices (MUTCDs) classified traffic incidents into three categories [7]. These are:

- Major traffic incident,

- Intermediate traffic incident, and

- Minor traffic incident.

Major Traffic Incident

Any traffic incident terms as the major incident if it takes more than 2 hours to clear the traffic [7]. Typical major traffic incidents are fatal traffic crashes, spill back of hazardous materials etc. So, proper traffic control devices should be implemented to reduce the impact on freeway traffic operations. An example of traffic incident management area signs is illustrated below in Figure 1.

Intermediate Traffic Incident

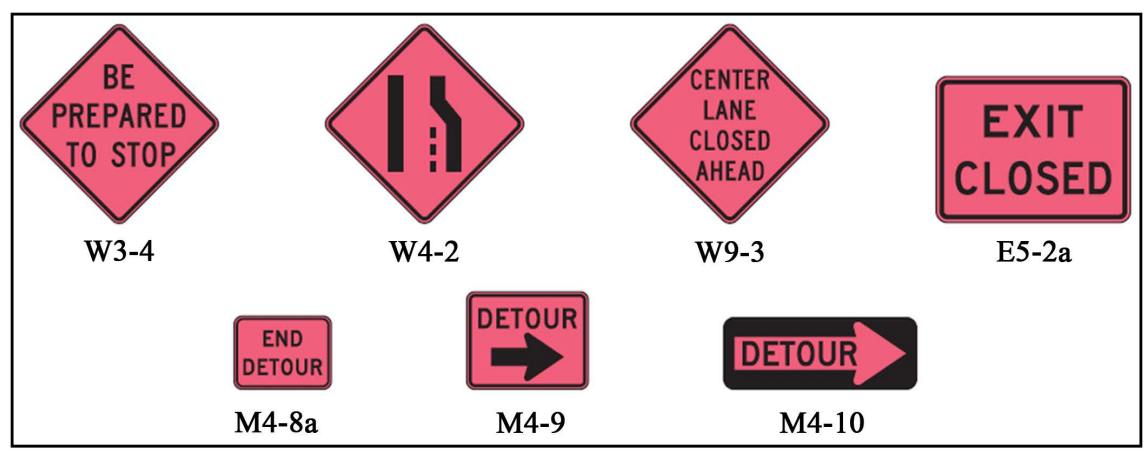

Figure 1. Common signs used in Traffic Incident Management, adapted from [7]. 
It represents incident duration 30 minutes to 2 hours [7]. So, full roadway closures needed for a short period to clear the traffic.

Minor Traffic Incident

It means incident duration less than 30 minutes [7]. So, traffic diversion may be needed for a short period.

In addition, according to traffic incident management handbook (2000), Cambridge Systematics develop a classification profile of traffic incidents based on incident type, location, and duration of the delay [8], illustrated in Figure 2.

\section{Problem Statement}

Freeway traffic incidents increases the congestion cost in terms of traffic delays, safety, and deterioration of the environment. Schrank et al. (2015) conducted a study and found traffic congestion trend is increasing significantly shown in Figure 3 \& Figure 4 [9].

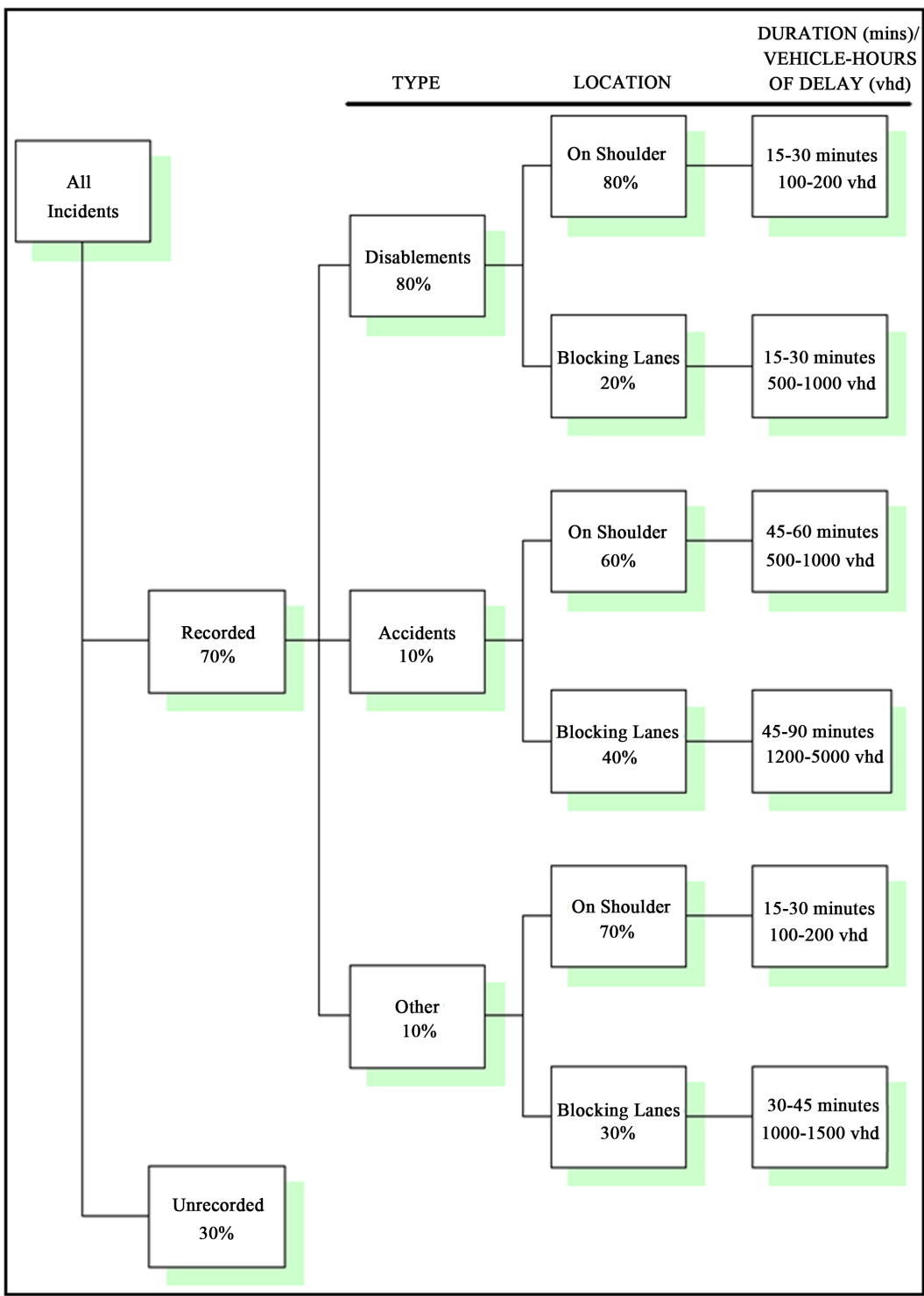

Figure 2. Reported incidents profile based on field data, adapted from [8]. 


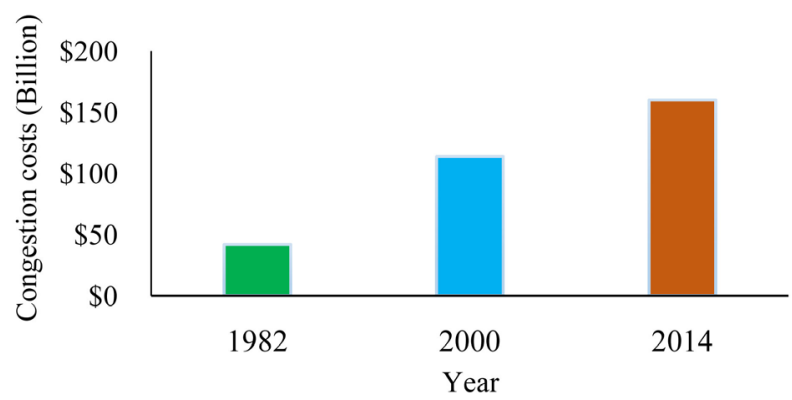

Figure 3. Trend of congestion cost in US, adapted from [9].

Trend of traffic congestion in the U.S. by city size (Hours of delay per auto commuter)

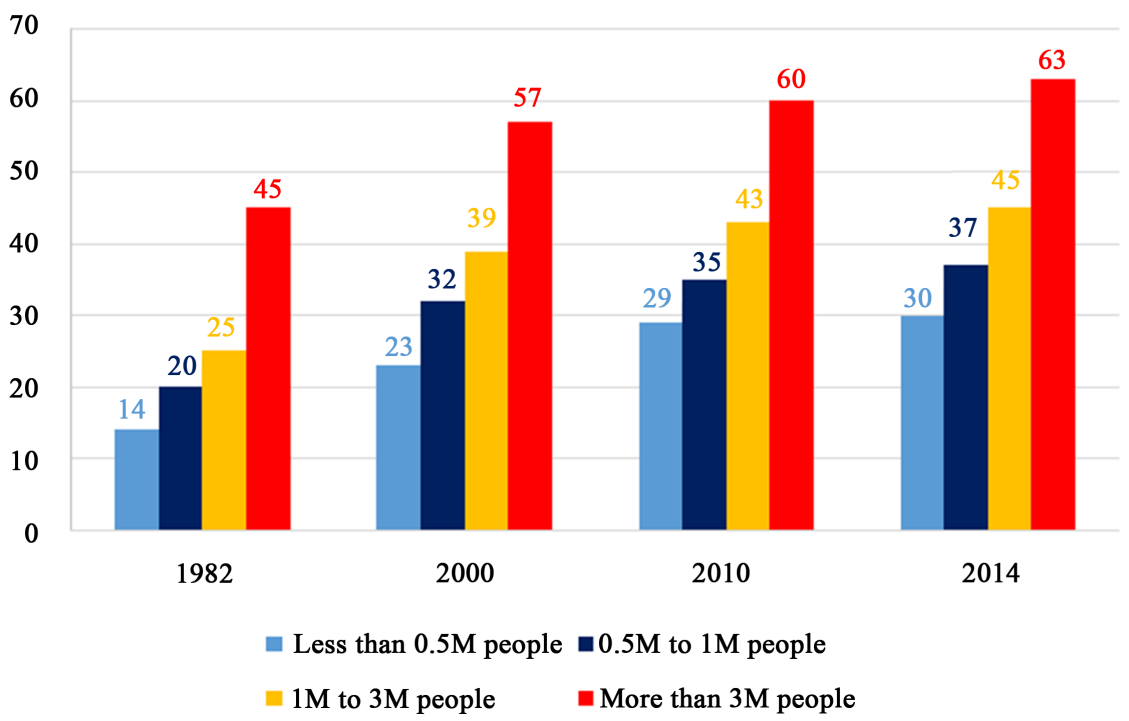

Figure 4. Trend of traffic congestion in the US by city size (Hours of delay per auto commuter), adapted from [9].

\subsection{Objectives}

The main goals of this study are:

- To summarize existing studies to identify the reasons for and impacts of the traffic incidents.

- To identify the gaps which are not yet addressed to improve the operational effectiveness of the freeway.

\subsection{Organization of the Paper}

- The first section of the paper provides the research background and objectives of the study.

- The second section of the paper summarizes past studies related to the incident detection algorithms, and the impacts of traffic incidents on freeway traffic operations.

- Finally, this paper concludes the crucial findings and recommendations for future research from the comprehensive literature review. 


\section{Literature Review}

This chapter summarizes existing peer-reviewed studies related to the freeway incidents and their impacts. The primary purpose of this chapter is to summarize past studies by answering the following two questions:

- What are the available practices to detect incident impacts on the freeway?

- What are the impacts of the traffic incidents on freeway traffic operations?

\subsection{Available Practices to Detect Incident Impacts on the Freeway}

The incident detection algorithm is an essential component to quantify the traffic congestion on freeway. Three most common metrics such as mean time-to-detect (MTTD), detection rate (DR), and false alarm rate (FAR) are used to assess the performance of the algorithm [10].

There are numerous studies on incident detection algorithms. These are discussed below in details.

\subsubsection{Comparative Algorithms}

Traffic parameter occupancy is used in comparative incident detection algorithms to identify the flow disruptions in the upstream and downstream at any adjacent stations. A good example of this types of algorithms is California decision tree and low pass filtering algorithm [11] [12]. California decision tree algorithm used average occupancy and traffic volume at regular interval 20s and 30s at each node. At every time step, several occupancy variables are identified and compared with preselected threshold values to determine the occurrence of an incident shown in Figure 5. However, algorithm 7 is only applicable for moderate to heavy traffic, not for low volume traffic.

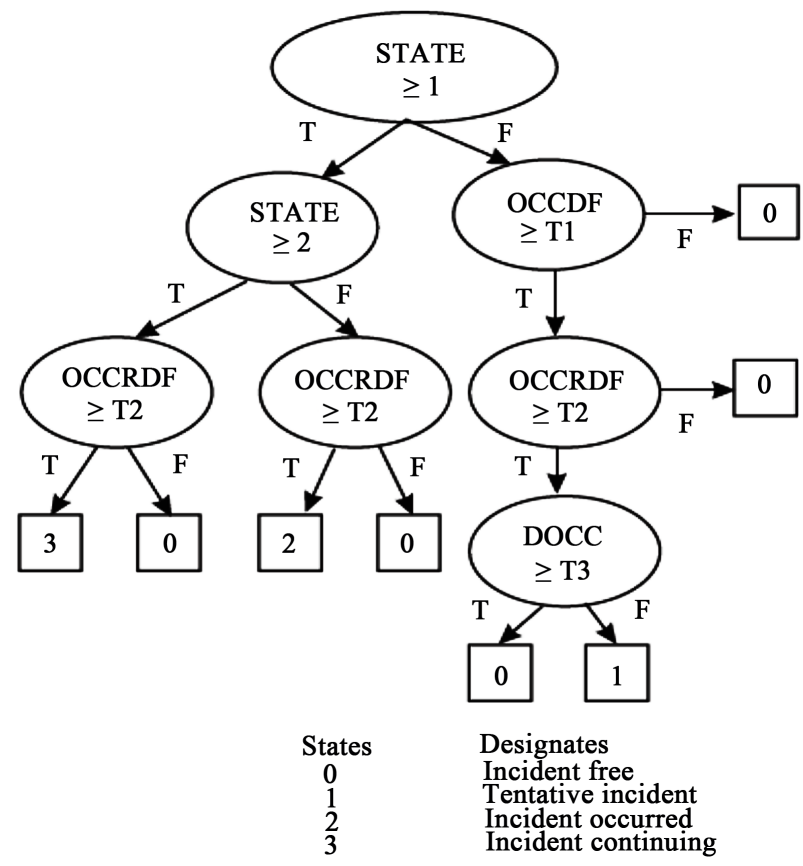

Figure 5. \#7 California decision tree algorithm (Source: [11]). 
Low pass filtering algorithm is developed by [12] to minimize the false alarm rate. This algorithm used average volume and occupancy at every $30 \mathrm{~s}$ interval. Authors evaluated the performance of the algorithm and results indicated 30\% $70 \%$ decrease in FAR. However, this algorithm is not capable to distinguish between rapidly evolving bottleneck congestion and incidents.

\subsubsection{Statistical Algorithms}

Statistical algorithms include Bayesian approach, SND (standard normal deviate) approach etc. Levin et al. (1978) developed a Bayesian algorithm to identify the incidents [13]. The algorithm uses occupancy as a variable in both $\mathrm{u} / \mathrm{s}$ and $\mathrm{d} / \mathrm{s}$ segments. Performance of this algorithm indicates $100 \% \mathrm{DR}, 0.0 \% \mathrm{FAR}$, and MTTD 3.9 minutes, but meantime to detect is more than another algorithm by almost 2.5 minutes.

Another study conducted by [14], proposed one algorithm based on a Bayesian approach, considering several variables between $\mathrm{u} / \mathrm{s}$ and $\mathrm{d} / \mathrm{s}$ detector stations. These variables include two traffic events, namely incident and congestion, and three traffic parameters, namely volumes, occupancies, and speed. The Bayesian network of this algorithm is shown in Figure 6.

The performance results of this algorithm indicate DR 92\%, FAR 0.087\%, and MTTD 175 s. However, some discrepancies in DR, FAR, and MTTD compared to others.

\subsubsection{Time Series and Filtering Algorithms}

Willsky et al., (1980) developed incident detection systems considering two algorithms [15]. In the proposed algorithm, authors considered three traffic variables, namely average velocities, flows, and densities. Performance of these algorithms was good, as there was no false alarms, $100 \%$ detection, and small response time over the range of flows $900-2000 \mathrm{cars} / \mathrm{hr} / \mathrm{ln}$. However, at low flows, both algorithms are unable to distinguish incidents from sensor biases.

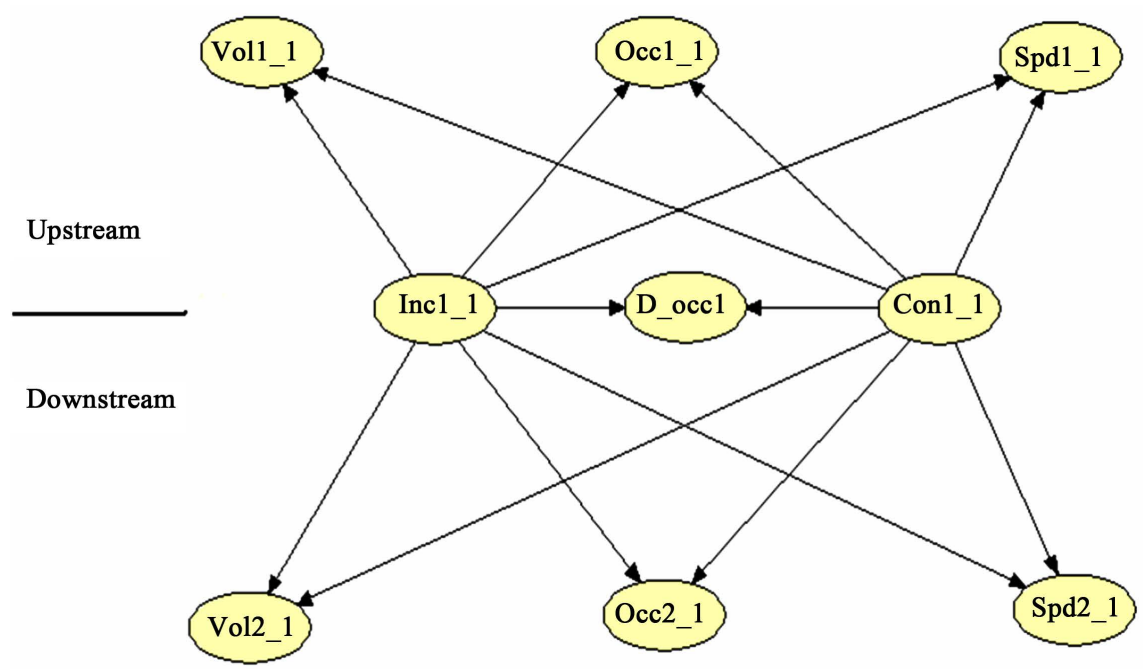

Figure 6. Freeway incident detection algorithm, A Bayesian approach (Source: [14]). 
There are also other time series and filtering algorithms, includes the autoregressive integrated moving average (ARIMA), and moving average (MA) [16] [17].

\subsubsection{Algorithms Considering Traffic Theory}

McMaster incident detection algorithm is the commonly traffic theory-based algorithm [18]. It considers flow, occupancy, and speed as a variable. The logic of the algorithm is illustrated in Figure 7.

\subsubsection{Advanced Incident Detection Algorithms}

[19] (Ghosh-Dastidar et al., 2003) Ghosh-Dastidar et al., (2003) proposed a wavelet-clustering model to identify incident on the freeway [19]. This model considers speed, volume, and occupancy as a variable. The logic of the model is shown in Figure 8. The test results indicate 100\% DR, 0.3\% FAR, and 35.6 seconds MTTD.

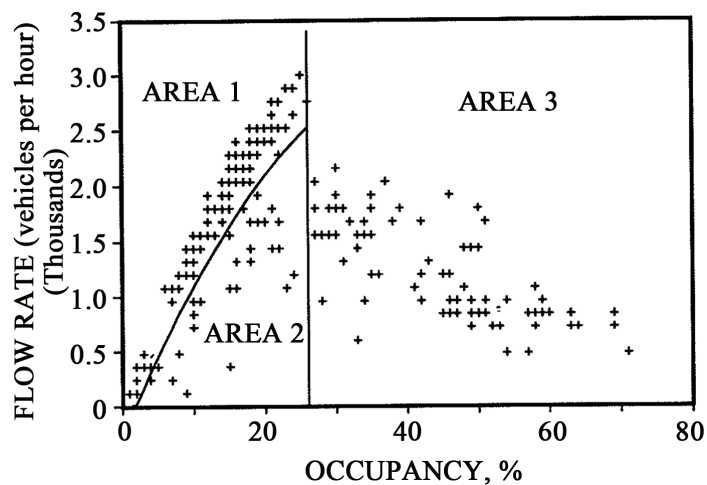

Figure 7. Three flow-occupancy areas shown with 30-sec data (Source: [18]).

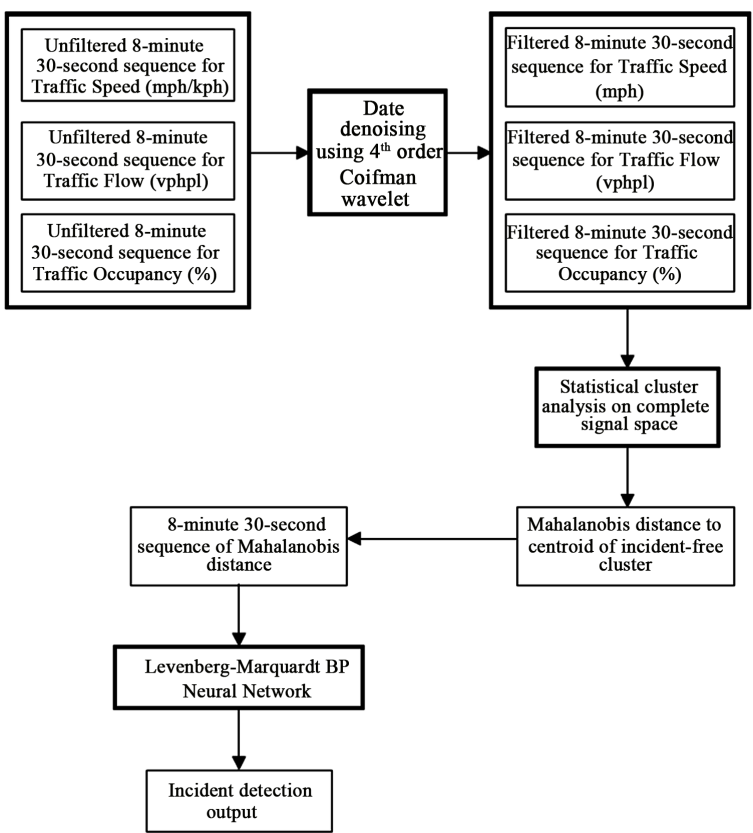

Figure 8. Wavelet-clustering algorithm for incident detection (Source: [19]). 
Karim et al. (2003) developed another fast automatic incident detection called wavelet energy algorithm [20]. The study used simulated data. The performance results of the algorithm showed DR 100\%, FAR 0.56\%, and MTTD less than 2 minutes. So, this model is not highly robust for field implementation.

Adeli et al. (2000) proposed wavelet-based denoising model using two types of data, simulated data and real-world data [21]. The results indicate DR 100\%, 95.2\%, and FAR $0 \%$ for simulated and filed data, respectively. But, the MTTD is 140 seconds which is higher. Also, the proposed model is applicable only for low to moderate volume traffic.

Jin et al. (2001), proposed another algorithm called constructive probabilistic neural network (CPNN) [22]. The study used volume, speed, and occupancy as a variable. The performance result indicates DR 92\%, and FAR $0.81 \%$.

Other advanced incident model include fuzzy logic algorithm [23], and artificial neural network (ANN) algorithm [10] [24].

In addition to the above classifications, there are also a set of models. Sullivan (1997) proposed a new model based on seven standard incident types, namely accidents and fires, abandoned vehicles, debris on the freeway, mechanical breakdown of the vehicles, tire problems, stalled vehicles, and other events like roadside fires, presence of pedestrian along freeways, etc. [2]. This prediction model is designed to estimate incident rate, severity, duration, and delays to assess the impacts of incidents. However, it didn't cover all types of incidents like traffic enforcement and maintenance work.

In an essence, advanced incident detection algorithm, particularly wavelet-clustering neural network model shows better performance than any other algorithms in comparison to results of the performance metrics and more importantly mean time to detect is less than almost 40 seconds. However, further research needs to fulfill all requirements.

\subsection{Impacts of Traffic Incidents}

There are several studies regarding the traffic incident impacts on freeway. Tavassoli Hojati et al. (2016) estimated the incident impacts on travel time reliability (TTR) on freeway using Tobit regression model [25]. The study concluded four variables significant significantly affect travel time reliability. However, the study didn't provide any validation of these results. Also, it didn't cover all other incidents like maintenance work and traffic enforcement.

Chen et al. (2003) evaluated the incident impacts on travel time, and concluded incident adds about 5 minutes to the median travel time [26]. However, the study didn't differentiate among the incidents which are more critical to evaluate the impacts of incidents as delay of travel time varies due to incident types.

Park et al. (2011) proposed an approach to estimate the incident impacts on TTR [27]. NTEGRATION simulation software has been used to obtain travel time and incident data. The study corridor was about 16-mile long segment on I-66 eastbound in Washington DC, USA. Three incident scenarios were consi- 
dered in this modeling. The authors concluded that TTR remarkably reduced during traffic incidents. However, the study used simulation data, not real data, and fixed incident duration which is not realistic.

Wright et al. (2015) also evaluate the incident impacts on TTR considering three types of incidents, namely shoulder, single lane and multi-lane incidents [28]. The study found all incidents induce to increase travel time reliability, compared to normal traffic conditions. Among them, multi-lane incident influences TTR and buffer index significantly, 205\% and 237\%, respectively. However, authors ignore other traffic incidents.

Skabardonis et al. (1997) evaluated the impact of incidents on traffic operations on a 9.2 miles segment in I-880 [29]. The study demonstrated how different types of traffic incidents such as accident, breakdown, and debris influence traffic operations. Total 2181 incidents were observed during the ninety-two 3-hr data-collection periods. The authors concluded the average duration of the incident was 25 minutes which increased the travel time of each traveler during that time. However, the study also didn't cover all types of incident events. Also, the authors addressed incidents impacts on travel time qualitatively not quantitatively.

Hojati et al. (2013) developed an approach to evaluate incident impacts considering three types of incidents, accidents, hazard, and abounded vehicles [30]. Authors used loop detectors data for a one year period. The results indicate the duration of incidents is $116,97,60$ minutes for crashes, hazards, and stationary vehicles which means impacts of incidents worsen the existing traffic operations. However, the study considered only three types of incidents. There are also other traffic incidents which can be incorporated in further research.

Incident affects the freeway capacity significantly. Several studies addressed this issue. Qin et al. (2001) developed an approach to estimate capacity reductions with the presence of accidents [31]. The results of this study indicate $63 \%$ and $77 \%$ capacity reduction for one lane and two lanes out of three lanes are blocked respectively. However, small capacity reductions cannot be estimated by using this method. The summary of the freeway traffic incident impacts on capacity reduction is illustrated in Figure 9.

Chimba et al. (2013) developed an approach to evaluate the duration of incidents on the freeway [33]. The study considered two types of incidents, namely abandoned and disabled vehicles. Descriptive analysis results showed average incident duration 57 minutes in Tennessee, 45 minutes in New Jersey, $31 \mathrm{mi}-$ nutes in Minnesota, and 60 minutes in North Carolina. These results indicate incidents are the main source of traffic congestion on the freeway.

Freeway traffic incidents can also affect the environment by increasing emission. S. Thomas et al. (2007) proposed an approach to evaluate the incident impacts on air pollution [34]. The study location was 16-mile-long along the I-80/94 freeway. Average incident duration was about 26 minutes. Authors compared the results of the emission with normal traffic condition. The result indicates incident induce $138 \%$ increase in CO. But, the study didn't represent 


\begin{tabular}{lcccc}
\hline & \multicolumn{4}{c}{ Close (\%) } \\
\cline { 3 - 5 } & Lanes & Shoulder & 1 Lane & 2 Lanes \\
\hline Goolsby, 1971 (21) & 3 & No data & 50 & 21 \\
1996 TCS Handbook (20) & 2 & 81 & 35 & 0 \\
& 3 & 83 & 49 & 17 \\
HCM 2000 (8) & 4 & 85 & 58 & 25 \\
1997 FM Handbook (3) & 3 & 74 & 51 & 21 \\
Qin and Smith, 2001 (22) & 3 & No data & 37 & 23 \\
Attica Tollway 2006-2007 & 2 & 79 & 44 & 0 \\
& 3 & 83 & 50 & 26 \\
& 4 & 87 & 59 & No data \\
\hline
\end{tabular}

Figure 9. Remaining capacity available with the presence of incidents (Source: [32]).

the results based on incident types. It just provides the overall impacts of incidents on the environment.

\section{Conclusions}

Proper management of freeway traffic operations is a vital issue as the number of traffic increases day by day, and lack of infrastructure development to support them. It results in congestion and makes the system worse if incidents present. Traffic incidents have several impacts, including that increased travel time results in extra delays and additional fuel consumption lead to higher emissions, and drivers stress increased driver aggressiveness. Transportation management center needs three types of information, namely location, types of incidents and duration of the delay to minimize the impacts of the traffic incidents. So, they can take the necessary traffic management control strategies to inform the drivers through traffic control devices.

Several studies have been done to develop some algorithms to detect traffic incident. Most of the researchers mainly focused on low to moderate volume traffic conditions and tested their algorithm. No one considers high volume traffic conditions i.e. oversaturated conditions which can be incorporated in further research. Another issue is with the reliability of these algorithms. As per MUTCDs and highway capacity manual (HCM), incident detection algorithms should be evaluated considering three parameters, namely DR, MTTD, and FAR. Most of the existing algorithms fail to fulfill all requirements. Some algorithm shows 100\% DR, 0\% FAR, but larger MTTD and some shows FAR 0\%, small MTTD, but DR approximately $90 \%$. Literature review revealed that only advanced incident detection algorithm, particularly wavelet-clustering neural network model shows better performance than any other algorithms in comparison to results of the performance metrics and more importantly mean time to detect is less than almost 40 seconds. However, further research needs to fulfill all requirements.

Few studies evaluated traffic incident impacts based on temporal and spatial distribution of the incidents. Incident impacts vary from time of day, location, 
and presence of special events. These factors should be considered in further research.

Quality of data is another issue like some records which indicate three lanes blocked by incidents, but there are only two lanes in the field. There are also other errors in the records. So, emerging vehicle technologies like connected vehicles can improve the data quality standard.

There are also some factors such as failures of communication and reader devices. These factors also need to consider in the incident detection algorithms.

In an essence, this study can serve as a reference to transportation agencies, researchers, and consultants involved in the assessment of freeway traffic operations based on traffic incidents to improve transportation network performance.

\section{Recommendations for Future Research}

Some study limitations are listed below, along with recommendations for potential future work:

- The study considered only freeway traffic operations. Evaluation of freeway and arterial interaction is recommended.

- Incorporation of connected vehicles should be considered to see the incident impacts on freeway operations.

\section{Conflicts of Interest}

The author declares no conflicts of interest regarding the publication of this paper.

\section{References}

[1] Skabardonis, A., Varaiya, P. and Petty, K. (2003) Measuring Recurrent and Nonrecurrent Traffic Congestion. Transportation Research Record: Journal of the Transportation Research Board, 1856, 118-124. https://doi.org/10.3141/1856-12

[2] Sullivan, E.C. (1997) New Model for Predicting Freeway Incidents and Incident Delays. Journal of Transportation Engineering, 123, 267-275. https://doi.org/10.1061/(ASCE)0733-947X(1997)123:4(267)

[3] Zhang, H. and Khattak, A. (2010) What Is the Role of Multiple Secondary Incidents in Traffic Operations? Journal of Transportation Engineering, 136, 986-997. https://doi.org/10.1061/(ASCE)TE.1943-5436.0000164

[4] Owens, N., Armstrong, A., Sullivan, P., Mitchell, C., Newton, D., Brewster, R. and Trego, T. (2010) Traffic Incident Management Handbook (No. FHWA-HOP-10-013). http://www.ops.fhwa.dot.gov/eto_tim_pse/publications/timhandbook/tim_handboo k.pdf

[5] Hall, R.W. (1993) Non-Recurrent Congestion: How Big Is the Problem? Are Traveler Information Systems the Solution? Transportation Research Part C: Emerging Technologies, 1, 89-103. https://doi.org/10.1016/0968-090X(93)90022-8

[6] Systematics, C. (2005) Traffic Congestion and Reliability: Trends and Advanced Strategies for Congestion Mitigation. Final Report, Texas Transportation Institute, College Station. http://ops.fhwa.dot.gov/congestion_report_04/index.htm

[7] Manual on Uniform Traffic Control Devices (MUTCDs) (2009) Federal Highway 
Administration (FHWA).

[8] Farradyne, P. (2000) Traffic Incident Management Handbook. Prepared for Federal Highway Administration, Office of Travel Management.

[9] Schrank, D., Eisele, B., Lomax, T. and Bak, J. (2015) 2015 Urban Mobility Scorecard.

[10] Thomas, K. and Dia, H. (2006) Comparative Evaluation of Freeway Incident Detection Models Using Field Data. IEE Proceedings of Intelligent Transport Systems, 153, 230-241. https://doi.org/10.1049/ip-its:20055015

[11] Payne, H.J. and Tignor, S.C. (1978) Freeway Incident-Detection Algorithms Based on Decision Trees with States. Transportation Research Record, 682, 30-37.

[12] Stephanedes, Y.J. and Chassiakos, A.P. (1993) Application of Filtering Techniques for Incident Detection. Journal of Transportation Engineering, 119, 13-26. https://doi.org/10.1061/(ASCE)0733-947X(1993)119:1(13)

[13] Levin, M. and Krause, G.M. (1978) Incident Detection: A Bayesian Approach. Transportation Research Record, 682, 52-58.

[14] Zhang, K. and Taylor, M.A. (2005) Towards Transferable Incident Detection Algorithms. Journal of the Eastern Asia Society for Transportation Studies, 6, 2263-2274.

[15] Willsky, A., Chow, E., Gershwin, S., Greene, C., Houpt, P. and Kurkjian, A. (1980) Dynamic Model-Based Techniques for the Detection of Incidents on Freeways. IEEE Transactions on Automatic Control, 25, 347-360. https://doi.org/10.1109/TAC.1980.1102392

[16] Ahmed, S.A. (1983) Stochastic Processes in Freeway Traffic Part I. Robust Prediction Models. Traffic Engineering \& Control, 24, HS-035775.

[17] Whitson, R.H., Burr, J.H., Drew, D.R. and McCasland, W.R. (1969) Real-Time Evaluation of Freeway Quality of Traffic Service.

[18] Persaud, B.N., Hall, F.L. and Hall, L.M. (1990) Congestion Identification Aspects of the McMaster Incident Detection Algorithm. Transportation Research Record, 1287, 167-175.

[19] Ghosh-Dastidar, S. and Adeli, H. (2003) Wavelet-Clustering-Neural Network Model for Freeway Incident Detection. Computer-Aided Civil and Infrastructure Engineering, 18, 325-338. https://doi.org/10.1111/1467-8667.t01-1-00311

[20] Karim, A. and Adeli, H. (2003) Fast Automatic Incident Detection on Urban and Rural Freeways Using Wavelet Energy Algorithm. Journal of Transportation Engineering, 129, 57-68. https://doi.org/10.1061/(ASCE)0733-947X(2003)129:1(57)

[21] Adeli, H. and Karim, A. (2000) Fuzzy-Wavelet RBFNN Model for Freeway Incident Detection. Journal of Transportation Engineering, 126, 464-471. https://doi.org/10.1061/(ASCE)0733-947X(2000)126:6(464)

[22] Jin, X., Srinivasan, D. and Cheu, R.L. (2001) Classification of Freeway Traffic Patterns for Incident Detection Using Constructive Probabilistic Neural Networks. IEEE Transactions on Neural Networks, 12, 1173-1187. https://doi.org/10.1109/72.950145

[23] Sheu, J.-B. (2002) A Fuzzy Clustering-Based Approach to Automatic Freeway Incident Detection and Characterization. Fuzzy Sets and Systems, 128, 377-388. https://doi.org/10.1016/S0165-0114(01)00141-5

[24] Ritchie, S.G. and Cheu, R.L. (1993) Simulation of Freeway Incident Detection Using Artificial Neural Networks. Transportation Research Part C: Emerging Technologies, 1, 203-217. https://doi.org/10.1016/S0968-090X(13)80001-0

[25] Tavassoli Hojati, A., Ferreira, L., Washington, S., Charles, P. and Shobeirinejad, A. (2016) Modelling the Impact of Traffic Incidents on Travel Time Reliability. 
Transportation Research Part C: Emerging Technologies, 65, 49-60. https://doi.org/10.1016/j.trc.2015.11.017

[26] Chen, C., Skabardonis, A. and Varaiya, P. (2003) Travel-Time Reliability as a Measure of Service. Transportation Research Record: Journal of the Transportation Research Board, 1855, 74-79. https://doi.org/10.3141/1855-09

[27] Park, S., Rakha, H. and Guo, F. (2011) Multi-State Travel Time Reliability Model: Impact of Incidents on Travel Time Reliability. 14th International IEEE Conference on Intelligent Transportation Systems, Washington DC, 5-7 October 2011, 2106-2111. https://doi.org/10.1109/ITSC.2011.6082874

[28] Wright, B., Zou, Y. and Wang, Y. (2015) Impact of Traffic Incidents on Reliability of Freeway Travel Times. Transportation Research Record: Journal of the Transportation Research Board, 2484, 90-98. https://doi.org/10.3141/2484-10

[29] Skabardonis, A., Petty, K., Bertini, R., Varaiya, P., Noeimi, H. and Rydzewski, D. (1997) I-880 Field Experiment: Analysis of Incident Data. Transportation Research Record: Journal of the Transportation Research Board, 1603, 72-79. https://doi.org/10.3141/1603-10

[30] Hojati, A.T., Ferreira, L., Charles, P. and Shobeirinejad, A. (2013) Quantifying the Impacts of Traffic Incidents on Urban Freeway Speeds. 36th Australasian Transport Research Forum, Brisbane, 2-3 October 2013.

[31] Qin, L. and Smith, B.L. (2001) Characterization of Accident Capacity Reduction (No. STL-2001-02). University of Virginia, Charlottesville.

[32] Prevedouros, P., Halkias, B., Papandreou, K. and Kopelias, P. (2008) Freeway Incidents in the United States, United Kingdom, and Attica Tollway, Greece: Characteristics, Available Capacity, and Models. Transportation Research Record: Journal of the Transportation Research Board, 2047, 57-65. https://doi.org/10.3141/2047-07

[33] Chimba, D., Kutela, B., Ogletree, G., Horne, F. and Tugwell, M. (2013) Impact of Abandoned and Disabled Vehicles on Freeway Incident Duration. Journal of Transportation Engineering, 140, Article ID: 04013013. https://doi.org/10.1061/(ASCE)TE.1943-5436.0000635

[34] Thomas, S. and Jacko, R. (2007) Stochastic Model for Estimating Impact of Highway Incidents on Air Pollution and Traffic Delay. Transportation Research Record: Journal of the Transportation Research Board, 2011, 107-115. https://doi.org/10.3141/2011-12 